\title{
Bow-tie optical antenna probes for single-emitter scanning near-field optical microscopy
}

\author{
Javad N Farahani ${ }^{1,4}$, Hans-Jürgen Eisler ${ }^{1,5}$, Dieter W Pohl ${ }^{1}$, \\ Michaël Pavius ${ }^{2}$, Philippe Flückiger ${ }^{2}$, Philippe Gasser ${ }^{3,6}$ and \\ Bert Hecht ${ }^{1,7}$ \\ ${ }^{1}$ Nano-Optics Group, National Center of Competence for Research in Nanoscale Science, \\ Institute of Physics, University of Basel, Klingelbergstrasse 82, CH-4056 Basel, Switzerland \\ ${ }^{2}$ Center of MicroNanoTechnology (CMI), École Polytechnique Fédérale de Lausanne (EPFL), \\ CH-1015 Lausanne, Switzerland \\ ${ }^{3}$ EMPA, Swiss Federal Laboratories for Materials Testing and Research, \\ Electronics/Metrology Laboratory, Überlandstrasse 129, CH-8600 Dübendorf, Switzerland
}

E-mail: hans.eisler@1ti.uni-karlsruhe.de and hecht@physik.uni-wuerzburg.de

Received 5 December 2006, in final form 15 January 2007

Published 23 February 2007

Online at stacks.iop.org/Nano/18/125506

\begin{abstract}
A method for the fabrication of bow-tie optical antennas at the apex of pyramidal $\mathrm{Si}_{3} \mathrm{~N}_{4}$ atomic force microscopy tips is described. We demonstrate that these novel optical probes are capable of sub-wavelength imaging of single quantum dots at room temperature. The enhanced and confined optical near-field at the antenna feed gap leads to locally enhanced photoluminescence (PL) of single quantum dots. Photoluminescence quenching due to the proximity of metal is found to be insignificant. The method holds promise for single quantum emitter imaging and spectroscopy at spatial resolution limited by the engineered antenna gap width exclusively.
\end{abstract}

\begin{abstract}
High-precision engineering of prototype devices that show function through their design and high complexity is a key target of applied nanoscale science and nanotechnology. Resonant optical antennas in the field of nano-photonics, with their architecture stimulated by their radio frequency counterparts, synergistically combine (i) electromagnetic field confinement and enhancement defined by the size of their feed gap width and (ii) impedance matching of optical waves mediated by the effective length of their antenna arms [1]. The control of sub-wavelength confined and enhanced optical fields pushes the limit in optical characterization [2-4],

\footnotetext{
4 Present address: Nanotechnology Group, ETH Zurich, Tannenstrasse 3, CH8092 Zurich, Switzerland.

5 Author to whom any correspondence should be addressed. Present address: Lichttechnisches Institut, Universität Karlsruhe (TH), Kaiserstrasse 12, D76131 Karlsruhe, Germany.

6 Present address: ETH Zurich, Elektronenmikroskopie-Zentrum ETH Zürich, HPT C 104, Wolfgang-Pauli-Str. 16, 8093 Zürich, Switzerland.

7 Author to whom any correspondence should be addressed. Present address: Nano-Optics and Bio-Photonics Group, Department of Experimental Physics 5, University of Würzburg, Am Hubland, D-97074 Würzburg, Germany.
}

manipulation [5-7], and optimization of single nanoscale light sources for information processing [8-11] on the nanometre scale. In particular, the impedance matching of optical waves opens an efficient pathway to transfer near-field information into the optical far-field, and vice versa [12].

In this paper, we present a strategy for designing bow-tie optical antennas at the apex of $\mathrm{Si}_{3} \mathrm{~N}_{4}$ atomic force microscopy (AFM) cantilever tips. We demonstrate that, even in this complex geometry, it is possible to control key antenna parameters such as overall length and width of the feed gap by focused-ion-beam milling. Merging well-established scanning probe technology with the concept of resonant optical antennas $[13,14]$ leads to a powerful new method of scanning optical microscopy in which an engineered optical hot spot is used as an optical probe [15]. We demonstrate the application of scanning optical antennas to the imaging of single quantum dots at room temperature. We show that the emission of individual quantum dots is enhanced when scanned across the antenna feed gap while concomitantly their excited-state lifetime is reduced. The field confinement, characterized by 


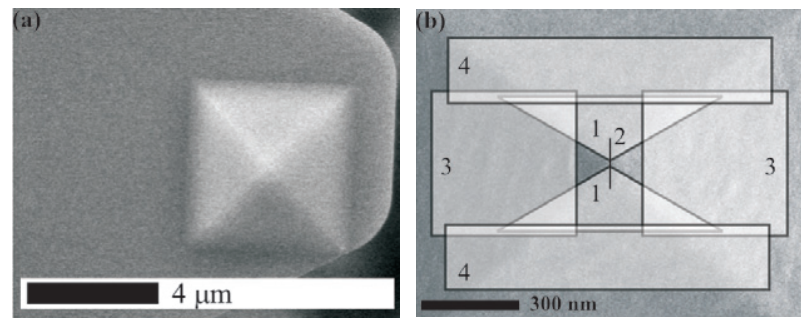

Figure 1. (a) SEM image of a clean AFM cantilever tip before metal evaporation; (b) FIB image of a fully coated AFM tip apex. The patches 1-4 indicate the area for FIB milling. (1) The first two triangular patches are milled, which defines the bow-tie antenna shape; the corner-to-corner distance of the triangles is $\approx 30 \mathrm{~nm}$. (2) A single line cut with an exposure time of $300 \mathrm{~ms}$ creates the antenna feed gap. (3) FIB milling of rectangular boxes sets the total length of the resonant optical antenna, $L$. (4) Removal of additional metallic patches.

the spot size found in the images, matches the dimension of the antenna feed gap well. Our method therefore qualifies for the imaging of single emitters at sub-wavelength spatial resolution only limited by the size of the antenna feed gap.

As an initial step for the preparation of antenna probes, pyramidal $\mathrm{Si}_{3} \mathrm{~N}_{4}$ AFM cantilevers (figure 1(a)) (DNP, Digital Instruments) were coated with a $40 \mathrm{~nm}$ thick aluminium film everywhere, including the tip. To this end, the cantilevers were mounted in an evaporation chamber. A tantalum boat was used as an evaporation source which was driven by resistive heating. The metal deposition rate, monitored by a quartz microbalance, was set to $2 \mathrm{~nm} \mathrm{~s}^{-1}$. At this rate the pressure during evaporation was $10^{-6}$ mbar. Characterization by scanning electron microscopy (SEM) after the metallization showed that more than $90 \%$ of all tips exhibited smooth and homogeneous metal coatings at the tip apex, while for the remainder the coating was disrupted by particles sticking to the tip. We found experimentally that, for metal film thicknesses $>40 \mathrm{~nm}$, the cantilevers start to bend, probably due to a mismatch between the thermal expansion coefficients of the silicon substrate and the $\mathrm{Al}$ thin film, rendering the cantilevers unusable for AFM.

Nicely coated tips were further processed by focused ion beam (FIB) milling. FIB milling was performed using a Dualbeam Nova 600 Nanolab or a Strata 235 DB from FEI, in which a vertical focused electron beam and a $52^{\circ}$ tilted focused Ga ion beam can be used alternately to characterize and modify the area of the substrate, respectively. Figure 1(b) shows a top-view scanning ion beam image of a representative tip. We created a well-defined nanometric metal structure by removing parts of the extended metal film, leaving behind the desired pattern. The best results have been achieved using an ion beam current of $10 \mathrm{pA}$. Figure 1(b) also depicts the sequence of onscale milling patterns that have been applied to create bowtie antennas at the apex of AFM tips. First, we defined the overall shape of the optical antenna by milling two triangles. The triangles were positioned at a finite distance to each other in order to ensure the presence of a metallic bridge at the apex. In a second step, the metal bridge was dissected carefully by a single ion beam line cut. This single line cut defined the width of the antenna feed gap. The gap is cut immediately to avoid focusing problems due to charging. A third milling step set the total length of the bow-tie optical antenna by removing the aluminium in two rectangular boxes positioned symmetrically with respect to the tip apex. The optical antenna engineering procedure was finalized by milling two more rectangles, as indicated in figure 1(b), in order to remove nearby metal. When considering the overall antenna length it is important to take into account the three-dimensional structure of the tip, which deviates strongly from a plane interface. The dimensions of the rectangles were chosen such that the distance between the antenna at the apex and the remaining metal film down the tip shaft was well below one wavelength.

The degree of control that was achievable using the outlined procedure is demonstrated by the series of three tips depicted in figures 2(a)-(f), in which the overall antenna length is varied between 120 and $200 \mathrm{~nm}$. As shown in the side views of the tips (figures 2(b), (d), (f)), variation of the dose by reducing the dwell time leads to different milling depths. The antenna of figure 2(a) actually rests on two $\mathrm{Si}_{3} \mathrm{~N}_{4}$ posts. Due to the finite depth of focus of the ion beam, this leads to a gap size $(\approx 50 \mathrm{~nm})$ that was larger than the nominal Ga-ion beam waist $(\approx 10 \mathrm{~nm})$. The silicon nitride posts may act as diffusion barriers, stabilizing the metal patches. For the other antennas, figures 2(c) and (e), the dose was optimized by decreasing the milling time to yield smaller feed gap widths $(\approx 25 \mathrm{~nm})$ while still making sure that the antenna arms were isolated from each other.

To prepare a suitable sample for microscopy, quantum dots in buffer solution $\left(10^{-9}\right.$ molar $)$ were spin-coated onto cleaned standard microscope cover slips. In a second step, to ensure that AFM scanning in contact mode did not result in pick up of quantum dots, we spun cast a dilute solution of poly-methylmetacrylate in toluene on top of the quantum dots. The coating resulted in a homogeneous $10 \mathrm{~nm}$ thick polymer film which covered the quantum dots almost completely. In AFM topography images of such films the locations of single quantum dots were hardly discernible. We assume no reorientation of the emission dipole moments of the single quantum dots during the experiment.

The setup has been described previously [16]. Briefly, it comprises a sample scanning confocal optical microscope combined with a tip-scanning AFM. The excitation light source was a pico-second, mode-locked $532 \mathrm{~nm}$ laser (GE-100, Time-Bandwidth) and pulse picker combination (model 350-160, Conoptics) operating at a repetition rate of $5 \mathrm{MHz}$. Confocal excitation and photoluminescence collection was performed by a high-numerical-aperture, oilimmersion microscope objective (Zeiss, Plan-Apochromat, $63 \times, 1.4 \mathrm{NA} \infty)$. After appropriate filtering, the collected light (photoluminescence emission around $585 \mathrm{~nm}$ ) was detected by a single-photon counting avalanche photodiode (SPAD, SPCM-AQR13, Perkin Elmer) connected to a timecorrelated single-photon-counting computer card (TimeHarp 200, PicoQuant, Berlin). For each single detected photon the arrival time was stored with high precision with respect to the start of the experiment as well as with respect to the previous laser pulse. This allowed us to reconstruct maps of the photoluminescence (PL) intensity as well as corresponding lifetime maps. Both types of information are important for an understanding of the interaction of single quantum dots with the optical antenna. The bow-tie antenna used in the experiments is shown in figures 2(a), (b). Its overall length 

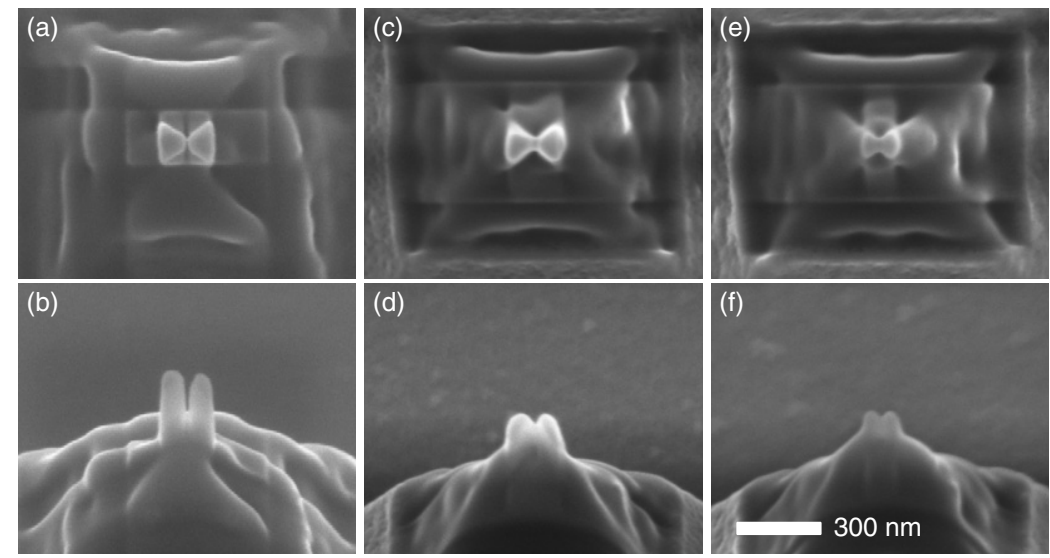

Figure 2. SEM images of three different bow-tie antenna lengths, $L$, in top-down and side-view projection: (a), (b) $L=170 \mathrm{~nm}$; (c), (d) $L=200 \mathrm{~nm}$; (e), (f) $L=120 \mathrm{~nm}$ - the antenna feed gap width varies between 25 and $50 \mathrm{~nm}$.
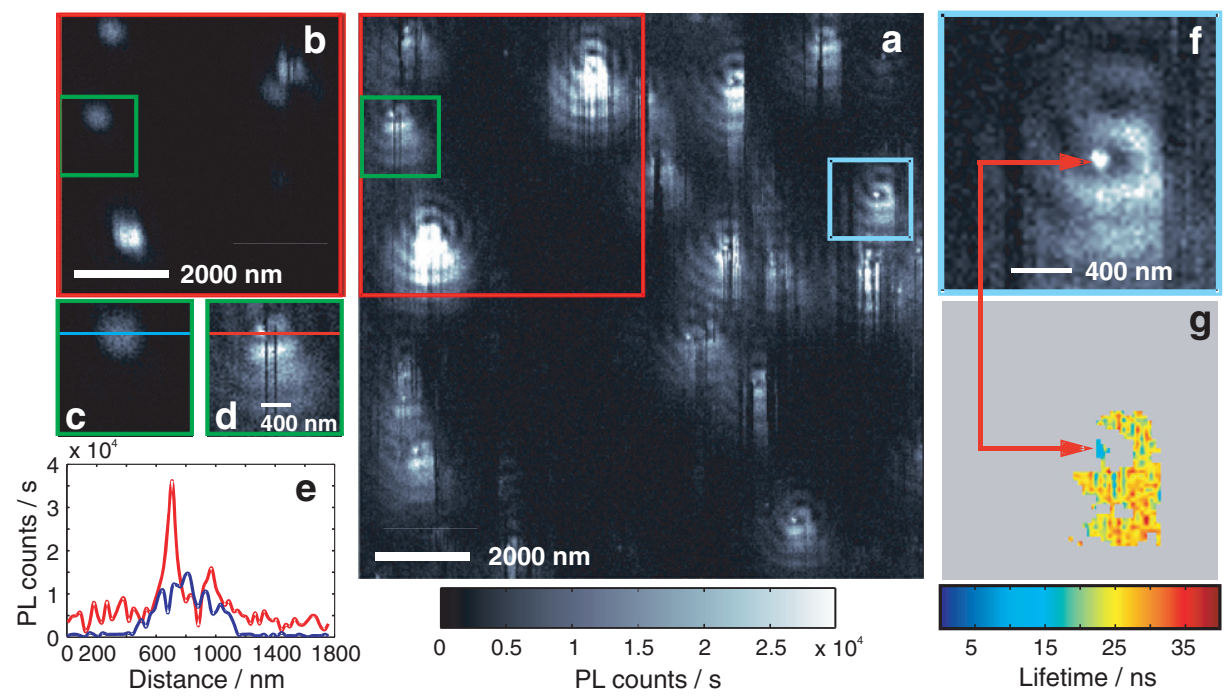

Figure 3. (a) $12 \mu \mathrm{m} \times 12 \mu \mathrm{m}$ near-field PL intensity image of single quantum dots. (b) PL confocal intensity image of single quantum dots from the region of (a) marked by the red rectangle; (c) extracted confocal PL intensity image of (b) marked by the green rectangle;

(d) extracted near-field PL intensity image of (a) marked by the green rectangle; (e) line profiles as indicated by the lines in (b) and (c) for detailed analysis; (f) PL intensity image of one single quantum dot marked by the blue rectangle in (a); (g) corresponding lifetime information of (f). Pixels with photon counts above an intensity threshold of 15 counts $\mathrm{ms}^{-1}$ are shown.

amounts to $170 \mathrm{~nm}$ with a feed gap width of $50 \mathrm{~nm}$. This is longer than the estimated half-wave antenna resonance length of $120 \mathrm{~nm}$, assuming an average dielectric constant (glass and $\mathrm{Si}_{3} \mathrm{~N}_{4}$ ) of the environment, and the first minimum of the antenna responsivity at about twice that length [16].

We present two sets of data recorded in different modes of operation. First, the antenna probe was positioned inside the laser focus at the sample surface using contact-mode AFM. Then the sample was raster-scanned while the relative position of the focal spot and antenna probe remained constant. Scanning of the sample eventually led to situations where isolated quantum dots were scanned beneath the optical antenna probe. Figure 3(a) shows a $12 \mu \mathrm{m} \times 12 \mu \mathrm{m}$ PL intensity map obtained in such an experiment.

In a second experiment the antenna probe was retracted and the same sample surface area was scanned again, yielding a confocal PL intensity image of single quantum dots (cf figure 3(b)).
Raster-scanning the quantum dot sample $12 \mu \mathrm{m} \times$ $12 \mu \mathrm{m}$ beneath the constantly illuminated optical bow-tie antenna resulted in a number of well-separated PL features comprising a small central PL spot accompanied by a slightly asymmetric interference pattern of constant fringe periodicity (see figure 3(a)). The distribution of the PL features resembles the two-dimensional distribution of photoactive quantum dots in the sample, as measured by scanning confocal optical microscopy (figure 3(b)). The asymmetry of the PL features is most probably caused by the fact that the AFM tip is positioned slightly off-centre with respect to the diffraction-limited laser spot. Vertical dark stripes in the high-intensity PL regions indicate the single-emitter nature of the quantum dots [17] as being the sources of the PL and show the direction of the slow scan axis.

As a reference for the size and shape of the PL intensity distribution in the focus, we use the $6 \mu \mathrm{m} \times 6 \mu \mathrm{m}$ confocal PL image (tip retracted) (figure 3(b)) of an area indicated by 
the red rectangle in figure 3(a). The confocal PL intensity map can be used as a reference, since the laser excitation intensity for this map and all other data had been kept constant at $1.8 \mu \mathrm{W} \mu \mathrm{m}^{-2}$, well below the saturation intensity of the quantum dots [16]. Comparison of a typical PL feature in the confocal PL image, figure 3(c), and the corresponding antenna modified PL feature, figure 3(d), clearly demonstrates two main features: (i) the PL response of the quantum dot interacting with the optical antenna is locally enhanced by a factor of three with respect to the peak of the diffractionlimited confocal spot and (ii) the full-width-at-half-maximum of the locally enhanced PL spot size matches the feed gap width of the optical bow-tie antenna $(\approx 50 \mathrm{~nm})$ as supported by the corresponding line cuts in figure 3(e). This unique observation holds true for most of the single quantum dot PL features in the sample area.

The localization of electromagnetic fields at the antenna feed gap can be utilized for optical imaging beyond the diffraction limit. The interaction of localized, enhanced electromagnetic fields with single emitters-here single CdSe/ZnS quantum dots - provides the possibility of gaining control over the fundamental opto-electronic response functions, such as the spontaneous emission rate of photons of the quantum system. The spontaneous emission rate is modified due to changes in the density of states of the photon-accepting photonic modes of the environment [18]. Creating a spatio-temporal optoelectronic hybrid structure in the weak coupling regime may lead to an enhanced spontaneous emission rate while the photoluminescence quantum yield stays unperturbed at near unity $[16,19]$. Analysing the lifetime information recorded simultaneously with the PL intensity images of a single quantum dot (see figures 3(f) and (g), respectively) shows that the PL enhancement is accompanied by a reduction in PL lifetime by a factor of approximately three. This observation indicates the birth of a super-emitter [16]. Furthermore, using the lifetime information, in the PL intensity image it is possible to differentiate between interference fringes due to constructive and destructive superposition of the back-scattered PL emission from the tip's side faces and the locally enhanced, central PL spot, since we exclusively observe a drastic reduction in the PL lifetime at the position of the enhanced central PL spot.

In conclusion, we have successfully implemented the concept of resonant optical antennas as a novel imaging tool for near-field microscopy using top-down nano-fabrication. We are able to precisely control the antenna parameters, such as the antenna arm length and the width of the antenna feed gap, and position such structures at the apex of an AFM tip. The interaction of an optical bow-tie antenna with a single quantum dot at ambient conditions confirms the character of locally enhanced and confined electromagnetic fields by design and proves optical resolution well beyond the diffraction limit of light.

\section{Acknowledgments}

The authors acknowledge H-J Güntherodt for continuous support. Financial support by the Swiss National Science Foundation is acknowledged via the National Center of Competence in Research (NCCR) in Nanoscale Science and a research professorship for one of the authors $(\mathrm{BH})$. One of the authors (H-JE) acknowledges individual funding through the Swiss Treubel-Award and acknowledges the award of the Heisenberg Fellowship sponsored by the Deutsche Forschungsgemeinschaft.

\section{References}

[1] Hecht B et al 2006 Resonant optical antennas and single emitters Advances in Nano-Optics and Nano-Photonics vol 1 Tip Enhancement ed S Kawata and V M Shalaev (Amsterdam: Elsevier)

[2] Kneipp K et al 1997 Phys. Rev. Lett. 781667

[3] Hartschuh A, Sanchez E J, Xie X S and Novotny L 2003 Phys. Rev. Lett. 90095503

[4] Knoll B and Keilmann F 1999 Nature 399134

[5] Novotny L, Bian R X and Xie X S 1997 Phys. Rev. Lett. 79645

[6] Xu H and Käll M 2002 Phys. Rev. Lett. 89246802

[7] Hallock A J, Redmond P L and Brus L E 2005 Proc. Natl Acad. Sci. USA 1021280

[8] Krenn J et al 1999 Phys. Rev. Lett. 822590

[9] Barnes W, Dereux A and Ebbesen T 2003 Nature 424824

[10] Maier S et al 2003 Nat. Mater. 222

[11] Michler P et al 2000 Science 2902282

[12] Pohl D W 2000 The Second Asia-Pacific Workshop on Near Field Optics (Beijing, Oct. 1999) ed M Ohtsu and X Zhu (Singapore: World Scientific)

[13] Schuck P J et al 2005 Phys. Rev. Lett. 9417402

[14] Mühlschlegel P et al 2005 Science 3081607

[15] Stöckle R M, Suh Y D, Deckert V and Zenobi R 2000 Chem. Phys. Lett. 318 131-6

[16] Farahani J N et al 2005 Phys. Rev. Lett. 95017402

[17] Nirmal M et al 1996 Nature 383802

[18] Purcell E M 1946 Phys. Rev. 69681

[19] Fisher B, Eisler H-J, Stott N and Bawendi M 2004 J. Phys. Chem. B 108143 